\title{
High Temperature Performances of CVD Single Crystal Diamond Detectors
}

\author{
R. Pilotti ${ }^{1}$, M. Angelone ${ }^{2}$, S. Loreti ${ }^{2}$, G. Pagano ${ }^{2}$, M. Pillon ${ }^{2}$, F. Sarto ${ }^{2}$, Marco Marinel- \\ $\mathrm{li}^{1}$, E. Milani ${ }^{1}$, G. Prestopino ${ }^{1}$, C. Verona ${ }^{1}$, G. Verona-Rinati ${ }^{1}$
}

Dipartimento di Ingegneria Industriale, Università di Roma "Tor Vergata"

Via del Politecnico 1, Roma, Italy

E-mail: riccardo.pilottilenea.it

2

ENEA, Centro Ricerche Frascati, UT-FUS

Via Enrico Fermi 45, Rome, Italy

In fusion applications (e.g. tokamaks) detectors must withstand a very harsh environment characterized by high temperatures and intense radiation fluxes. Conventional solid state detectors (e.g. silicon) cannot be operated at high temperatures and present serious dose limit. Among the possible alternatives to silicon, diamond based detectors are very promising because of their outstanding properties such as radiation hardness, high thermal conductivity and wide band gap. Diamond detectors were proposed as neutron detectors for the Radial Neutron Camera (RNC) and for the Tritium Breeding Module (TBM) of ITER. In the latter the detector working temperature can be up to $400{ }^{\circ} \mathrm{C}$. A few studies, so far have been performed to study the behaviour of diamond detectors at high temperatures. In the present paper the performances of single crystal diamond detectors operated at high temperatures are reported. An innovative detector layout was studied which is based on a mechanical contact avoiding the use of critical components such as glue, welding, etc. A $500 \mu \mathrm{m}$ thick single crystal diamond plate with platinum electrical contacts was tested reaching temperatures up to $240{ }^{\circ} \mathrm{C}$, its I-V and $\mathrm{C}-\mathrm{V}$ characteristics were measured at increasing temperatures. The results demonstrated that the proposed innovative layout presents very interesting behaviour and stable response at high temperatures. Furthermore, a commercial detector was tested at temperatures up to $180{ }^{\circ} \mathrm{C}$ while operated in current mode using a beta source. The signal to noise ratio vs. temperature resulted acceptable up to about $170{ }^{\circ} \mathrm{C}$.

Keywords : diamond detectors, high temperature, harsh environment, tokamak diagnostics

First EPs Conference on Plasma Diagnostics - $1^{\text {st }}$ ECPD

14-17 April 2015,

Villa Mondragone, Frascati (Rome) Italy

${ }^{1}$ Speaker
${ }^{\odot}$ Copyright owned by the author(s) under the terms of the Creative Commons Attribution-NonCommercial-ShareAlike Licence. 


\section{Introduction}

Fusion technologies have been characterized by impressive developments but some issues are still present. Dedicated and/or special materials are needed for diagnostic systems to be operated in future tokamaks (e.g. ITER) and, among them, diamond is one of the most promising materials due to its outstanding properties: wide band gap $(5.5 \mathrm{eV})$, very high resistivity $\left(>10^{15}\right.$ $\Omega \mathrm{cm})$, high carrier mobility $\left(4500 \mathrm{~cm}^{2} \mathrm{~V}^{-1} \mathrm{~s}^{-1}\right.$ for electrons and $3800 \mathrm{~cm}^{2} \mathrm{~V}^{-1} \mathrm{~s}^{-1}$ for holes), and high breakdown field (>10 $\left.\mathrm{MV} \mathrm{cm}^{-1}\right)$ [1].

Diamond can withstand very harsh environments like those present inside the Test Blanket Modules (TBM) in ITER [2][3]. TBMs are elements designed to study the tritium production and are characterized by intense neutron flux (up to $4 * 10^{14} \mathrm{n} \mathrm{cm}^{-2} \mathrm{~s}^{-1}$ ) and very high temperatures $\left(300-500{ }^{\circ} \mathrm{C}\right)$ [4]. Diamond based detectors were proposed as on-line tritium monitor for TBMs in ITER [5][6].

Several issues are to be faced and many problems have to be solved to realize a diamond detector capable to properly operate at high temperature. The starting point of this work is represented by the results reported in [7]. In [7] the behavior of commercial diamond detectors produced by Diamond Detectors Limited (DDL) company [8] was studied. A diamond detector 50 $\mu \mathrm{m}$ thick was irradiated by a ${ }^{241} \mathrm{Am}$ alpha source and operated with $25 \mathrm{~V}$ bias voltage. The position of the $5.5 \mathrm{MeV}$ alpha peak in the pulse height spectrum (PHS) vs. temperature was studied by locating the detector on top of an electrical heater. The acquisition chain was composed by H.V. unit Ortec 556, charge amplifier ORTEC 142, shaping amplifier ORTEC 570 and multichannel analyser (MCA) ORTEC 918. In Figure 1 the experimental results are presented. Figure 1 shows that commercial diamond detectors can be operated up to about $175{ }^{\circ} \mathrm{C}$. Beyond this limit a drop of detector's resolution and a clear shift of the peak centroid is clearly visible.

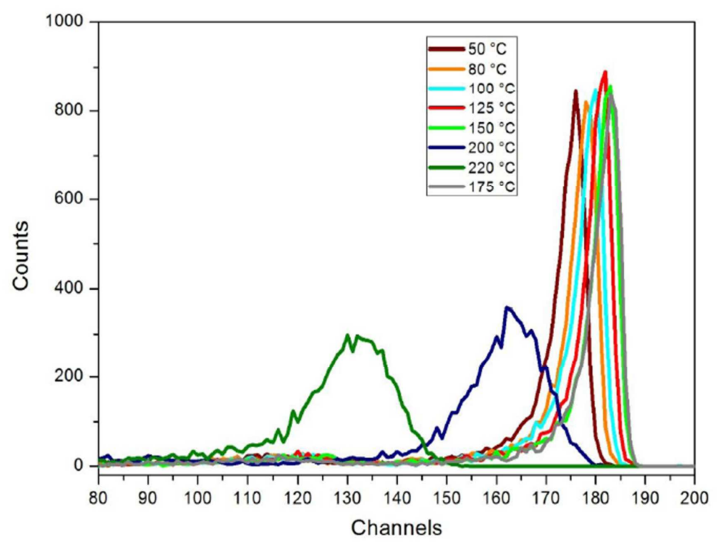

Figure 1: ${ }^{241}$ Am peaks vs temperature

The main problems with commercial detectors are related to the presence of welding and conductive glues as well as the use of cables and connectors not optimized for high temperature applications [9]. A dedicated effort is also to be devoted to the study of the best metal contact to be used with diamond, since at the interface metal-diamond a Schottky barrier is formed $(\sim 1$ $\mathrm{eV})$ whose amplitude and stability with the temperature depends on the type of the used metal. The best metal seems to be $\mathrm{Ag}$ whose barrier is stable up to about $800{ }^{\circ} \mathrm{C}[10]$.

In this paper an innovative diamond detector which does not use any welding and/or glue is presented and the results up to about $240{ }^{\circ} \mathrm{C}$ are reported. Furthermore, since the TBM environment is characterized by a very intense neutron flux, the diamond detector should be operat- 
ed in current mode. This was never studied before at high temperature. The experimental results obtained operating, for the first time, a commercial diamond detector at high temperature (180 ${ }^{\circ} \mathrm{C}$ ) are also presented.

\section{A new diamond detector based upon mechanical layout}

As seen in previous studies [9], the presence of glues and welding in diamond's detector affect the performances of the detectors when operated at high temperatures $\left(>170{ }^{\circ} \mathrm{C}\right)$. In this work an innovative diamond detector lay-out based only on a mechanical contact without the need to use conductive paste or glues as well as welding is presented.

The used diamond film is a Single Crystal Diamond (SCD), $4.5 \times 4.5 \mathrm{~mm}$ side and $0.5 \mathrm{~mm}$ thickness. The diamond film was first annealed at $500{ }^{\circ} \mathrm{C}$ in vacuum for $1 \mathrm{hr}$ and then two $\mathrm{Pt}$ contacts ( $3 \mathrm{~mm}$ in diameter and $100 \mathrm{~nm}$ thick) were deposited on top of both faces of the diamond film by sputtering technique. The diamond detector was thus sandwiched between two metal plates, the so called upper and lower electrodes. The upper electrode consists of a metal plate in mechanical contact with the external case (ground). The lower plate acts as an electrode being connected to the central wire of a standard SMA connector by means of a small metallic spring. The lower metal plate is hosted in a Teflon disc, properly shaped as to insulate it from the external case. Figure 2 reports the mounting scheme of the MCD detector [7].

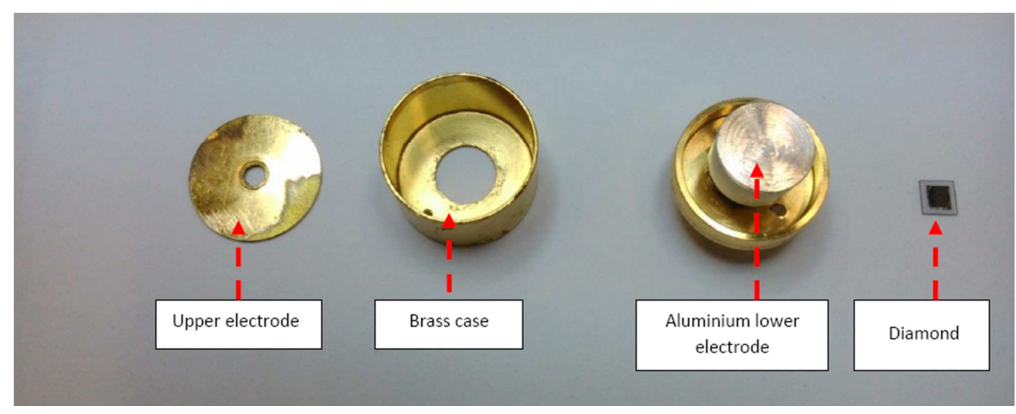

Figure 2: picture of the mechanical diamond detector

\section{Experimental results}

To investigate the response and behaviour of the MCD detector a series of different tests at various temperatures were performed.

In a first series of tests, the density current versus voltage $(\mathrm{J}-\mathrm{V})$ and the capacitance vs. voltage $(\mathrm{C}-\mathrm{V})$ characteristics were measured. The experimental results are shown in Figure 3 (a) and Figure 3 (b). The J-V characteristic was performed using a Keithly 6517A picoammeter and the C-V measurements using the Agilent 4263B LCR meter. It is ought to be stressed that in these preliminary tests, for connecting the detector to the front-end electronics a standard cable was used, this cable is not optimized nor recommended for high temperature applications. Figure 3 (a) shows the J-V characteristic measured at various temperatures. The current density is obtained by dividing the current by the electrode surface which is $0.09 \mathrm{~cm}^{2}$. The data reported in Figure 3 (a) clearly show the presence of a Schottky barrier on both side of the detector [11]. The curves are rather symmetric indicating that the detector is well realized. 

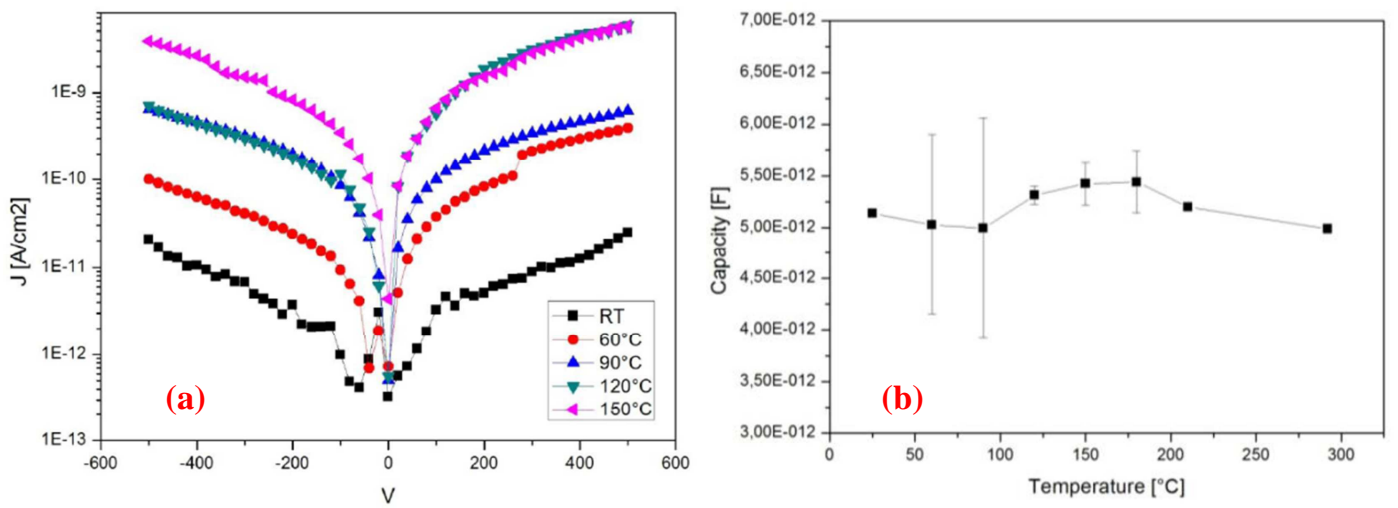

Figure 3: (a)J-V characteristic measured at various temperatures for the mechanical diamond detector; (b) detector's mean capacity measured at various temperature

A preliminary test of the MCD detector under neutron irradiation was also performed at the Frascati Neutron Generator (FNG), FNG produces up to $10^{11} \mathrm{n} \mathrm{s}^{-1} 14 \mathrm{Mev}$ neutrons by the D-T fusion reaction [12]. The detector was operated with 400 bias voltage. The acquisition chain was composed by H.V Ortec 556, charge amplifier ORTEC 142, shaping amplifier ORTEC 570 and multichannel analyser (MCA) ORTEC 918. In Figure 4 the pulse shape spectrum (PHS) acquired at room temperature and at $100{ }^{\circ} \mathrm{C}$ is shown. Fig. 4 shows that the ${ }^{12} \mathrm{C}(\mathrm{n}, \alpha){ }^{9} \mathrm{Be}$ peak produced in carbon by $14 \mathrm{MeV}$ neutrons and acquired at $100{ }^{\circ} \mathrm{C}$ overlaps the peak acquired operating the detector at room temperature. At higher temperatures an increasing shift between the two peaks was observed as well as an increasing effect of the thermal noise.

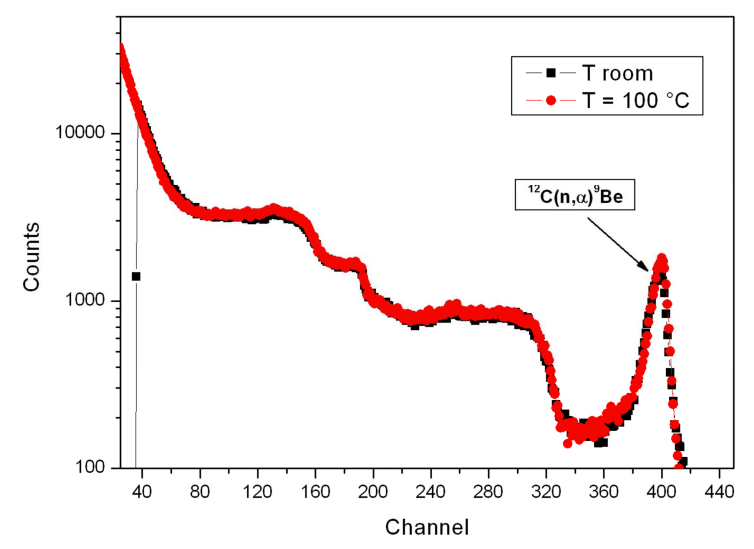

Figure 4:PHS spectrum acquired under neutron irradiation at room temperature and $100{ }^{\circ} \mathrm{C}$

\subsection{Operation in current mode}

The TBM environment is characterized by an intense neutron flux, intense gamma flux and high temperatures. The strong neutron and gamma fluxes (up to $3 * 10^{14} \mathrm{n} \mathrm{cm}^{-2} \mathrm{~s}^{-1}$ for neutrons and an order of magnitude lower for gammas) arises some serious concerns about the possibility to operate neutron flux monitors in counting mode, since counting rate of several tens up to several $\mathrm{MHz}$ are expected. Thus, it is important to study the feasibility of current mode operation at high temperature for the neutron detectors to be located in TBMs.

Current mode is routinely used with diamond detectors for X-ray and UV detection; however these measurements are performed at room temperature. At high temperature the increasing of dark current produced by thermal excitation lead to a degradation of the Signal/Noise ratio 
$(\mathrm{S} / \mathrm{N})$. In this paper the first attempt ever reported of using a diamond detector operating in current mode at high temperature is presented. For this study a commercial $500 \mu \mathrm{m}$ thick diamond detector produced by DDL company was used. The detector was connected by a mineral cable (MI) to the ammeter and the current was measured with a Keithley 6487 Picoammeter. The "1 C CAc 30 Si" used MI cable is produced by the Thermocaox company and it is designed for harsh environment (up to $800^{\circ} \mathrm{C}$ ) and very fast signals. The detector was operated with a bias voltage of $500 \mathrm{~V}$ while the tests were performed at various temperatures starting from $20{ }^{\circ} \mathrm{C}$, using a beta source $1 \mathrm{MBq}$ Sr-90. The experimental results presented in Figure 5 demonstrate that diamond detectors can be operated in current mode at high temperatures. The maximum operating temperature available for commercial diamond detectors is close to $180{ }^{\circ} \mathrm{C}$, at this temperature the measured $\mathrm{S} / \mathrm{N}$ ratio is about 5.5. Above $180{ }^{\circ} \mathrm{C}$ the $\mathrm{S} / \mathrm{N}$ ratio drops and the detector does not work properly.

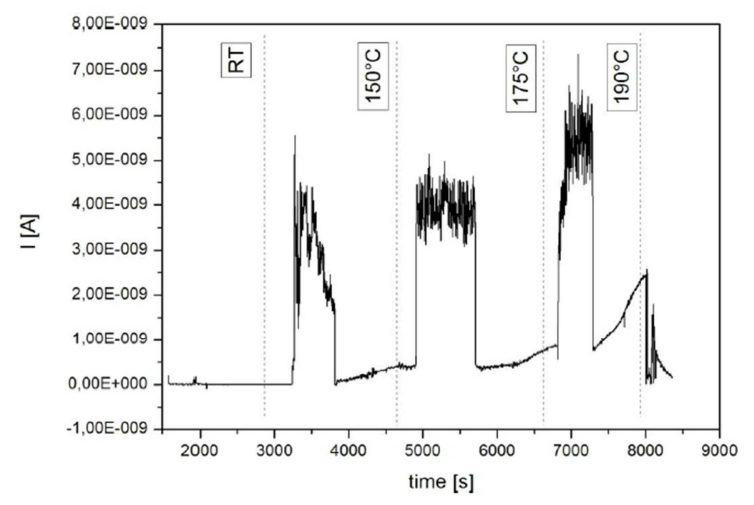

Figure 5: acquired current for temperature up to $190{ }^{\circ} \mathrm{C}$ with the Sr-90source

\section{Discussion and conclusion}

The experimental results reported in this work while confirming the capability of diamond detectors to operate at high temperatures, once more point out the need to improve diamond detectors layout to reach a stable operating temperature of at least $300{ }^{\circ} \mathrm{C}$. MCD layout represents an important improvement because avoiding the use of critical elements (e.g. welding and conductive paste/glue) allows to increase the working temperature. Optimizations of the mechanical layout is going to be studied: the use of special conductive glues for high temperature applications $\left(1000^{\circ} \mathrm{C}\right)$ as well as a comparative study of different metals for electrical diamond contacts are under investigation. It is important to point out that the reported results were obtained using standard cables (but not for current mode) and connectors which are not recommended for operation at temperatures higher than $160-180{ }^{\circ} \mathrm{C}$. It is difficult then to to say how much their use affected the reported results. For the above reason, the diamond detectors designed to operate at high temperatures need to be equipped by mineral cables (MI) and special connectors able to operate at high temperature. Mineral cables are designed to operate up to $800{ }^{\circ} \mathrm{C}$ while metaloxide connectors (MOC) able to withstand up to $400{ }^{\circ} \mathrm{C}$ are available on the market. Their use is presently under implementation and a first prototype equipped both with MI and MOC was just realised. Preliminary measurements allowed to operate the detector under $14 \mathrm{MeV}$ neutron irradiation up to $230{ }^{\circ} \mathrm{C}$ with promising performances (Figure 6). 


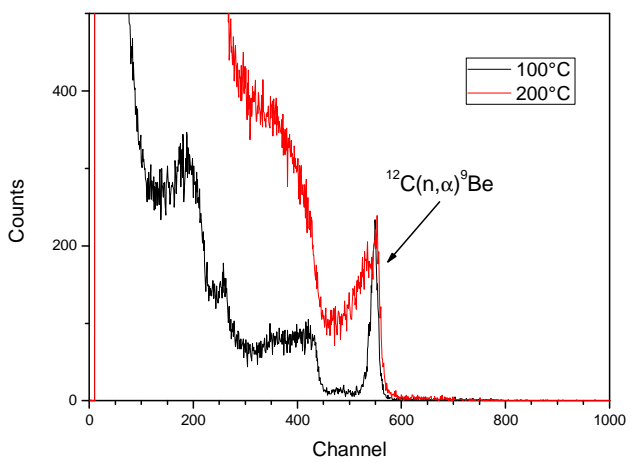

Figure 6: Response functions measured at $100^{\circ} \mathrm{C}$ and $200^{\circ} \mathrm{C}$ respectively with a prototype diamond detector equipped with $\mathrm{MI}$ and MOC.

The experimental results presented in this paper showed that diamond detectors are very promising for fusion diagnostic applications at high temperatures. The new mechanical layout discussed in the paper is very interesting because avoiding the use of critical elements (e.g. welding and conductive paste/glue) allows the increase of the working temperature. More studies to further optimize the mechanical layout foresee the use of different mechanical detectors design and the use of MI and metal-ceramic connectors able to withstand $400{ }^{\circ} \mathrm{C}$.

\section{References}

[1] S. Koizumi, C. Nebel, and M. Nesladek, "Physics and applications of CVD diamonds", Wiley-Vchc Verlag GmbH \& Co., 2008.

[2] M. Angelone, et al.," Radiation hardness of a polycrystalline chemical-vapor-deposited diamond detector irradiated with 14 MeV neutrons", Rev. Sci. Instr. 77, 2006.

[3] M. Pillon, et al.," "Radiation tolerance of a high quality synthetic single crystal CVD diamond detector irradiated by 14.8 MeV neutrons" Journ. Appl. Phys 104, 2008.

[4] L. Petrizzi and R. Villari, "Final report on HCLL: TBM design, integration and analysis. Neutronic evaluation of TBM in ITER machine", ENEA Fusion Division Nuclear Fusion Technologies 2005.

[5] M. Angelone, et al., "Behaviour of LiF covered single crystal diamond detectors operated at high temperature under neutron irradiation", 2013 IEEE Nuclear Science Symposium, IEEE Cat. Num. CFP13NSS-DVD,Seul, Oct. 2013.

[6] M. Pillon, et al., "Development of On-Line tritium monitor based upon artificial diamond for fusion applications", IEEE transactions on nuclear science, vol. 58, n. 3, 2011.

[7] R. Pilotti, "Development of artificial diamond detectors operating at high temperature for tritium detection in breeding blanket of thermonuclear fusion reactors", Master Thesis, May 2014, Università degli Studi "La Sapienza" Roma .

[8] A. Galbiati, "Performance of Monocrystalline Diamond Radiation Detectors Fabricated Using TiW, Cr/Au and a Novel Ohmic DLC/Pt/Au Electrical Contact", IEEE TNS vol. 56, n. 4, 2009.

[9] M. Angelone, et al., "Spectrometric performances of monocrystalline artificial diamond detectors operated at high temperature",IEEE transactions on nuclear science, vol. 59, n. 5, 2012.

[10] K. Ueda, K. Kawamoto, T. Soumiya, and H. Asano, "High-temperature characteristics af Ag and Ni/diamond Schottky diodes," Diamond \& Related Materials,n. 38, pp. 41-44, 2013.

[11] D. Evans, "Diamond metal contact : interface barrier and real time characterization", J. Phys : Condens. Matter, vol. 21, p. 364223, 2009.

[12] M. Angelone, et al., "Absolute experimental and numerical calibration of the $14 \mathrm{MeV}$ neutron source at the frascati Neutron Generator",Rev. Sci. Instrum., n. 67, p. 2189, 1996. 